\title{
The change and maintenance effectiveness of persistence training regarding the treatment of laboratory-induced and naturally occurring depression
}

\author{
JACK R. NATION and JOHN B. COONEY \\ Texas A\&M University, College Station, Texas 77843
}

\begin{abstract}
A study was conducted to evaluate the therapeutic effectiveness of persistence training as a model for treating depression. Subjects were female college students who were exposed to experimental manipulations to induce learned helplessness or who reached multiple-depression criteria on the MMPI and Beck Depression Inventory. Helpless and depressed subjects were treated with either response-contingent continuous reinforcement or partial reinforcement (persistence training) on a human shuttle apparatus. Following therapy, all subjects encountered recurring failure during an extinction phase. The findings showed that continuous and partial reinforcement schedules were equally effective in therapy, resulting in improved performances for both helpless and depressed subjects. There was evidence, however, that the treatment gains were less for depressed subjects than for helpless subjects, regardless of the type of therapy schedule used. With respect to maintenance of change, it was found that both helpless and depressed subjects persisted longer in the face of successive nonreinforcements (extinction) following partial reinforcement therapy as opposed to continuous reinforcement therapy. Further, there was evidence that depressed subjects persisted less in extinction than did helpless subjects, but only when therapy had occurred under conditions of continuous reinforcement.
\end{abstract}

Persistence training refers to a four-stage program for treating depression, the features of which presume to increase maintenance of behavioral change (cf. Nation \& Massad, 1978). The hallmark of the therapy model is the inclusion of a partial reinforcement phase (Stage 3). From a purely logical standpoint, persistence training has a firm foundation, resting on the findings from basic infrahuman learning research that shows subjects given partial reinforcement in acquisition evidence more resistance to extinction than do comparable subjects given continuous reinforcement (see Robbins, 1971, for a review). The idea, regarding therapy for depressive symptoms occasioned by situational stress, is that intermittent success (reinforcement) and failure (nonreinforcement) outcomes for criterion nondepressive behaviors will enhance maintenance of change by fostering a tolerance for a nonsupportive environment. That is, when a patient encounters protracted periods of adversity extratherapy, the coping skills acquired during therapy are more likely to survive (persist in the face of extinction), given that initial changes were achieved with partial reinforcement.

To date, experimental inquiries relevant to establishing the validity and reliability of the therapeutic effects associated with the persistence training technique have

Requests for reprints should be sent to Jack R. Nation, Department of Psychology, Texas A\&M University, College Station, Texas 77843. been restricted to populations in which the depression is mild and characterized as laboratory induced (e.g., Nation, Cooney, \& Gartrell, 1979; Nation \& Massad, 1978). Such a narrow approach to documenting the efficacy of the treatment program is understandable when one realizes that persistence training evolved from the literature on another laboratory phenomenon known as learned helplessness (cf. Abramson, Seligman, \& Teasdale, 1978; Maier \& Seligman, 1976). Moreover, it is likely that the generation of any new intervention procedure should involve a substantial period of basic research under controlled conditions, prior to implementation (Agras, Kazdin, \& Wilson, 1979). This sort of systematic progression in therapy research is highly desirable, in that it permits careful delineation of essential treatment components and inhibits premature extrapolations to applied settings.

Nevertheless, some confirmation of the beneficial treatment effects common to persistence training probably is needed with a more traditional population of depressives. The importance of this particular issue derives from conflicting reports in the area of reversing learned helplessness and depression. Klein and Seligman (1976), for instance, found that forced exposure to success (soluble concept-identification problems) alleviated the performance deficits associated with either laboratory-induced learned helplessness or actual depression. An independent experiment by Kilpatrick-Tabak 
and Roth (1978), however, found that similar cognitive reversal manipulations were therapeutic for learned helplessness subjects, but ineffective with depressed subjects. The controversy created by these discrepant findings is exceedingly important and has implications not only for the learned helplessness theory of depression (Abramson et al., 1978) but for persistence training as well. Perhaps the demonstrated effectiveness of persistence training is in fact peculiar to treating laboratory depression.

The purpose of this study was to answer such questions concerned with the appropriateness of persistence training as a therapy device for ameliorating naturally occurring depression. Specifically, the experiment described herein compared the relative effectiveness of traditional therapy procedures (continuous reinforcement) and persistence training (partial reinforcement) with respect to (1) the reversal of learned helplessness or alleviation of depression and (2) the subsequent maintenance of change in the face of successive experiences with nonreinforcement (extinction).

\section{METHOD}

Subjects were 60 Texas A\&M University female undergraduates enrolled in introductory psychology. The subjects were selected from a pool of 1,176 introductory students and were assigned to one of two categories using multiple criteria. Forty students were classified as nondepressed by virtue of (1) scoring below the top quartile on the D-scale of the MMPI and (2) scoring 9 or below on the Beck Depression Inventory (BDI). Twenty students were classified as depressed by virtue of (1) scoring above the top quartile on the D-scale of the MMPI and (2) scoring 13 or above on the BDI. Meeting criteria in all cases was sequentially dependent; that is, MMPI scores were first obtained for all 1,176 students, and subsequently, subjects were administered the BDI to determine their eligibility for participation in the experiment.

\section{Apparatus and Materials}

Pretreatment. A series of six five-dimensional conceptidentification problems served as pretreatment stimuli. Details concerning the dimensions used, order of presentation, and so on, are provided elsewhere (Nation et al., 1979).

Therapy. The therapy apparatus was a shuttlebox identical to that described for the therapy phase (task-dissimilar subjects) of the Nation et al. (1979) experiment. Movement of the peg to the extreme left turned off a moderately aversive tone, as described in Nation et al. Depending on the location of a selector switch, continuous reinforcement (tone termination), 50\% partial reinforcement, or extinction training could be administered.

Extinction. The apparatus used during therapy was again used in extinction.

Mood scales. Three 10-point scales were used to provide an index of sadness, frustration, and personal competency. Each scale asked the subject to indicate "the way you feel at this moment." High scores on the sadness and personal competency scales corresponded to "extremely sad" and "very incompetent," respectively, and low scores on the frustration scale corresponded to "very frustrated."

\section{Procedure}

Subjects were administered the MMPI under group conditions. Upon arrival at the laboratory, subjects were seated at a desk and administered the BDI. On the basis of the combined scores, a subject was assigned to one of four nondepressed groups, was assigned to one of two depressed groups, or was dismissed. Eligible subjects completed the mood scales before starting the experiment and again following each of the three phases.

Pretreatment. Subjects in the nondepressed category experienced different manipulations according to group assignment. Subjects in the no-treatment group (ND-NoT) received exactly the same experiences as those of the same group in Nation et al. (1979). That is, neither success nor failure was experienced during this initial phase, because subjects simply looked at the pretreatment stimuli. Group ND-SOL received six soluble problems, and two insoluble groups (ND-IN-CRF, ND-IN-PRF) received the same helplessness-induction manipulation experienced by the corresponding groups in the Nation et al. study; that is, none of the six problems had solutions.

Subjects in the depressed category (Group D-CRF and Group D-PRF) received the same pretreatment experiences as subjects in the no-treatment group.

Therapy. Three nondepressed groups (ND-NoT, ND-SOL, ND-CRF) and one depressed group (D-CRF) received 35 response-contingent continuous reinforcement trials as therapy. Conversely, one nondepressed group (ND-IN-PRF) and one depressed group (D-PRF) received 5 continuous reinforcement trials followed by 30 partial reinforcement trials as therapy (persistence training). On reinforcement trials, moving the peg to the extreme left solved the problem (turned off the tone). On nonreinforced trials, the tone would not terminate regardless of the number of criterion shuttle responses performed. Details concerning instructions, maximum tone length, intertrial interval, signaled presentation, and so on, can be found in Nation et al. (1979).

The dependent measures obtained on the shuttlebox task were latency (defined as the latency from trial onset to completion of the criterion behavior), number of failures (number of trials on which subjects failed to make the criterion response), and number of responses (the number of times the subject made the criterion response during a given trial). The measures were used to (1) document the occurrence of helplessness and depression and (2) show the reversal effects of the different therapy schedules.

Extinction. Upon completion of therapy training, subjects in all six groups were given 40 extinction trials. The procedure for all extinction trials was identical to that for nonreinforced trials during therapy; that is, the shuttle responses were not effective in terminating the tone. The instructions were as described for the extinction phase of the Nation et al. (1979) experiment. The same dependent measures used for therapy were again used in extinction to index persistence.

\section{RESULTS}

\section{Mood Data}

The analysis performed on the BDI scores showed that subjects categorized as depressed had significantly higher scores than subjects categorized as nondepressed $[F(5,54)=62.99, p<.01]$. Group means were as follows: $\mathrm{D}-\mathrm{CRF}=17.3, \mathrm{D}-\mathrm{PRF}=16.9, \mathrm{ND}-\mathrm{NoT}=4.8$, ND-SOL $=4.6, \mathrm{ND}-\mathrm{IN}-\mathrm{CRF}=4.8$, and ND-IN-PRF $=4.9$.

The three separate repeated-measures analysis of variance (ANOVA) tests performed on the sadness, frustration, and personal competency measures showed that depressed subjects (D-CRF, D-PRF) and helplessness subjects (ND-IN-CRF, ND-IN-PRF) exhibited more pronounced negative moods following pretreatment than did the remaining subjects. Therapy training (regardless of type) was generally effective in reversing the negative 
moods induced in the laboratory (ps $<.05)$. The negative effect associated with nonlaboratory depression was less reactive to therapy manipulations; that is, the depressed subjects continued to report greater sadness and frustration than the other subjects at the end of therapy (ps <.05). Reversal effects were obtained for both helpless subjects (ND-IN-CRF, ND-IN-PRF) and depressed subjects (D-CRF-, D-PRF) on the personal competency measure, however. Following extinction, all groups showed significant increases in levels of reported sadness ( $\mathrm{ps}<.05)$ and frustration $(\mathrm{ps}<.01)$. The personal competency measure yielded differential group results. Interestingly, Group D-PRF failed to show a significant increase in perceived personal incompetency following extinction and felt more competent than Group D-CRF, which did experience a significant increase in perceived personal incompetency following extinction (all ps $<$ .05). Similar findings were obtained with the comparisons involving Groups ND-IN-CRF and ND-IN-PRF (ps < .05). These data suggest that partial reinforcement in therapy (persistence training) operates to immunize helpless and depressed persons against the negative influence of protracted nonreinforcement, at least regarding reported levels of perceived personal incompetency.

\section{Behavioral Data}

Therapy. Analysis of the three dependent measures used for therapy showed pronounced and reliable behavioral depression in the helplessness groups (ND-IN-CRF, ND-IN-PRF) and the depression groups (D-CRF, D-PRF) relative to the remaining groups (ND-NoT, ND-SOL). It was further shown that continuous reinforcement and partial reinforcement resulted in significant improvement, both in terms of reversing helplessness and in terms of alleviating depression. However, there was evidence that both types of schedules failed to achieve a complete amelioration of depression; that is, Groups D-CRF and D-PRF were inferior to all other groups at the end of therapy.

The repeated-measures ANOVA performed on the latency measure yielded significant main effects for groups $[F(5,56)=9.94, p<.01]$ and blocks $[F(6,322)$ $=146.68, \mathrm{p}<.01]$. Newman-Keuls post hoc comparisons of group means showed that Groups D-CRF and D-PRF had longer latencies than Groups ND-IN-CRF and ND-IN-PRF, which had longer latencies than Groups ND-NoT and ND-SOL. Inspection of interaction means showed that while the helplessness and depression groups did improve significantly over blocks of therapy trials, the depression groups were still slower to respond on the last block than were the remaining four groups, which were not different from each other. The blocks effect was due to shorter latencies across trials.

The analysis of the failures data showed the same group differences noted for latency $[\mathrm{F}(5,56)=14.69$, $\mathrm{p}<.01]$. A significant interaction $[\mathrm{F}(30,322)=6.90$, $\mathrm{p}<.01$ ] permitted comparisons that showed that
Groups D-CRF, D-PRF, ND-IN-CRF, and ND-IN-PRF failed more on Block 1 than Groups ND-NoT and ND-SOL. On Blocks 2 and 3, Groups D-CRF and D-PRF failed more than the remaining four groups, which did not differ from each other. None of the groups differed on Blocks 4-7. The fact that therapy resulted in fewer failures across trials was revealed in the report of a significant blocks effect $[F(6,322)=83.21, p<.01]$.

Regarding the number-of-responses data, the only finding of interest was the report of a significant interaction $[\mathrm{F}(30,322)=7.94, \mathrm{p}<.01]$. Comparisons involving all means showed depression and helplessness effects were obtained early in therapy but disappeared in all groups except D-CRF by the end of therapy. The greater number of responses on the part of D-PRF subjects relative to D-CRF subjects likely reflects a design confound due to the increased opportunity in the former case to make multiple responses on nonreinforced trials.

Extinction. The extinction data showed substantially greater persistence for subjects given partial reinforcement during therapy (Groups D-PRF and ND-IN-PRF) relative to subjects given continuous reinforcement in therapy (Groups D-CRF, ND-IN-CRF, ND-NoT, and ND-SOL). While there were no differences between the groups receiving partial reinforcement (persistence training), there was some indication that depressed subjects were less persistent than nondepressed subjects following therapy experiences with continuous reinforcement.

Due to terminal therapy differences on the latency and number-of-responses measures, an Anderson (1963) rate transformation was performed on these two measures for extinction. This procedure permits a more realistic assessment of maintenance of behavioral change independent of degree of change initially. The analyses of the three dependent measures used for extinction showed similar results, with the strongest effects obtained for the number-of-responses (rate) measure. In the interest of space, detailed information concerning extinction responding is limited to this rate measure.

The extinction rates (number of responses) for all six groups are depicted in Figure 1. The repeated-measures ANOVA performed on this measure showed significant main effects for groups $[F(5,56)=12.84, p<.01]$ and blocks $[F(7,376)=68.74, p<.01]$. Comparisons of group means indicated that groups D-PRF and ND-INPRF, which were not different from each other, were more persistent than Group ND-IN-CRF, which was more persistent than Groups D-CRF, ND-NoT, and ND-SOL. The blocks effect was simply due to decreasing rates over trials.

A significant Groups by Blocks interaction was also found $[F(35,376)=5.13, p<.01]$. Post hoc analyses indicated that no group differences appeared on the first two blocks. The superiority of Groups D-PRF and ND-IN-PRF relative to remaining groups emerged by 


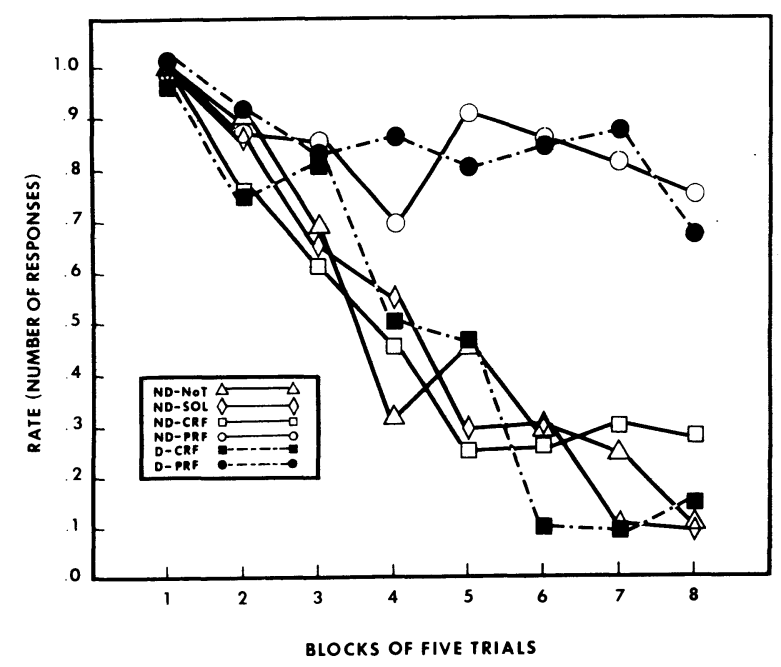

Figure 1. Extinction rates (number of responses) for the nondepressed groups (ND-NoT, ND-SOL, ND-CRF, ND-PRF) and the depressed groups (D-CRF, D-PRF) across blocks of five trials.

Block 4, and this pattern continued for remaining blocks. Interestingly, Group D-CRF was inferior to all other groups on Block 6 and was inferior to Group ND-IN-CRF on Block 7.

\section{DISCUSSION}

The results of this experiment show that negative affect and psychomotor retardation are symptomatic of both learned helplessness and depression, with the effects being more pronounced for depression: The finding of performance deficits associated with naturally occurring depression would seem to temper recent criticisms regarding the hypothesized parallel between learned helplessness and reactive depression. Specifically, it has been suggested that the deficits in instrumental responding that are common to learned helplessness are not typical of depressed persons (Lobitz \& Post, 1979). Clearly, our data show that such deficits do appear in depressed persons and thereby further the argument that helplessness and depression are mutually determined phenomena.

With respect to the efficacy of persistency training (Nation \& Massad, 1978), the findings from this study show that partial reinforcement is as effective as continuous reinforcement at alleviating depressive symptoms, even though neither schedule is successful in lifting depression completely. While other reward parameters might occasion different results, our inability to achieve total amelioration is consistent with at least one other report (viz., Kilpatrick-Tabak \& Roth, 1978). More important findings in this study, perhaps, relate to the maintenance issue associated with behavioral change. Persistence training (partial reinforcement therapy) produced dramatically greater immunization against recurrent failure (nonreinforcement) than did continuous reinforcement, both for helpless subjects and for depressed subjects. The depression results are of particular interest, in that more compelling evidence than has been previously available is now provided that persistence training is a valid clinical tool. The beneficial maintenance features associated with persistence training are even more impressive when one considers our finding that depressed persons treated with continuous reinforcement reacted more negatively during extinction than did comparably treated nondepressed subjects. Ostensibly, persistence training was able to overcome the tendency for depressed subjects to be more devastated by failure (i.e., Groups D-PRF and ND-IN-PRF did not differ during extinction).

The suggestion has been made that persistence training inhibits the onset of helplessness by virtue of forcing an attribution for failure outcomes to unstable factors (Seligman, Note 1). That is, intermittent success and failure experiences may foster an expectancy that reinforcement does occur eventually, if relevant behaviors continue to be executed. Whether or not such attributions actually are responsible for previous and present findings, or whether such findings are due to an Amsel-type frustration effect (cf. Nation \& Massad, 1978; Nation et al., 1979), the advance for behavior therapy is absolute; namely, greater maintainance of change can be achieved via scheduleinduced persistence.

\section{REFERENCE NOTE}

1. Seligman, M. E. P. Personal communication, May 1979.

\section{REFERENCES}

Abramson, L. Y., Seligman, M. E. P., \& Teasdale, J. D. Learned helplessness in humans: Critique and reformulation. Journal of Abnormal Psychology, 1978, 87, 49-74.

Agras, W. S., Kazdin, A. E., \& Wilson, G. T. Behavior therapy: Toward an applied clinical science. San Francisco: Freeman, 1979.

ANDERSon, N. H. Comparison of different populations: Resistance to extinction and transfer. Psychological Review, 1963, 70, 162-179.

Kilpatrick-TABaK, B., \& Roth, S. An attempt to reverse performance deficits associated with depression and experimentally induced helplessness. Journal of Abnormal Psychology, 1978, 87, 144-154.

Klein, D. C., \& Seligman, M. E. P. Reversal of performance deficits and perceptual deficits in learned helplessness and depression. Journal of Abnormal Psychology, 1976, 89, 11-26.

Loвitz, W., \& Post, R. D. Parameters of self-reinforcement and depression. Journal of Abnormal Psychology, 1979, 88, 33-41.

Maier, S. F., \& Seligman, M. E. P. Learned helplessness: Theory and evidence. Journal of Experimental Psychology: General, 1976, 105, 3-46.

Nation, J. R., Cooney, M. B., \& Gartrell, K. E. Durability and generalizability of persistence training. Journal of Abnormal Psychology, 1979, 88, 121-136.

Nation, J. R., \& Massad, P. Persistence training: A partial reinforcement procedure for reversing learned helplessness and depression. Journal of Experimental Psychology: General, 1978, 107, 436-451.

Roвbins, D. Partial reinforcement: A selective review of the alleyway literature since 1969. Psychological Bulletin, 1971, 76, 415-431.

(Received for publication June 5, 1980.) 